\title{
Cuidar de sí: una tecnología para los niños y niñas de Colombia*
}

\author{
Juan Pablo Suárez Bonilla**
}

Resumen. Este artículo presenta el cuidado de sí como una tecnología viable en el escenario educativo colombiano. Para esto hace un recorrido por el concepto, pasando por las valoraciones de Michel Foucault, Carol Gilligan y Nell Noddings quienes, por medio de sus propuestas incluso antagónicas, despiertan el interés por dicho tema, dejando claro que es una idea que se debe revisar, revitalizar y actualizar en la formación de los niños y las niñas del país y de la ciudadanía en especial, con el fin de cambiar el absurdo maltrato físico, ataque sexual y privación de los derechos de los menores en el territorio nacional. Finalmente, presenta las tecnologías del cuidado como unas prácticas de la libertad asociando la propuesta del pensador francés con las

* El presente artículo de reflexión hace parte de los referentes teóricos de la investigación sistematización del plan de vida del Colegio del Rosario de Santo Domingo curso 10C, año 2007; indagación desarrollada como requisito para obtener el título de Magíster en Desarrollo Educativo y Social, programa del convenio CINDE/Universidad Pedagógica Nacional.

** Magíster en Desarrollo Educativo y Social de la Fundación Centro Internacional de Educación y Desarrollo Humano (CINDE)/Universidad Pedagógica Nacional. Licenciado en Filosofía y docente investigador de la Universidad de San Buenaventura, sede Bogotá. Estudiante de Doctorado en Ciencias Sociales Niñez y Juventud CiNDE/Universidad de Manizales. Dirección electrónica de contacto: jpsuarez@academia.usbbog.edu.co 
estrategias presentadas por Noddings, animando el ambiente educativo a su reconocimiento y aplicación.

Palabras clave. Cuidado de sí, tecnologías del yo, prácticas de la libertad, modelar.

Summary. In this article, the care of the self is presented as a feasible technology in the Colombian educative scenario. The report presents the concept before mentioned in the light of Michel Foucault, Carol Gilligan and Nell Noddings view, who through their contradictory proposals show an interest for analyzing, improving and actualizing the care of the self concept in the educative context. This proposal is focused on girls and boys of the country and specially the citizenship, aimed at changing the absurd physical mistreatment, sexual abuse, and the deprivation of the minor's rights in the country. Finally, this assertion presents the technologies of the self as free practices associating Michel Foucault's proposal with the strategies presented by Noddings, encouraging the educational field to its recognition and application in the classroom.

Keywords. The care of the self, Technologies of the self, free practices, model.

\section{Introducción}

Pensar en asegurar los derechos y condiciones óptimas a niños y niñas en los actuales contextos sociales, no puede ser una tarea aplazada. Es urgente en el panorama nacional que todos los ciudadanos/as tomen cartas en el asunto para, de alguna manera, frenar los maltratos y atropellos con que se ha afectado a este importante sector de la población; para ello es fundamental sentirse partícipe en la construcción del entramado, como un actor que toma parte en la conformación de una sociedad justa, equitativa y plural en la 
que todos caben, pero también todos aportan. Desde esta perspectiva es fundamental abrir la escuela, la Universidad y todo centro educativo, para que su acción toque los sectores de la sociedad y de esta manera los individuos se sientan a la vez cuidadores unos de otros, con total entereza, responsabilidad y reciprocidad, con el fin de evitar la naturalización de la violencia que sobre estos indefensos seres se ha ensañado con absurda demencia, dañando sus cuerpos, interioridad, mirada, esperanza, presente y futuro. En este sentido la sociedad debe lanzar un grito continuo, constante y desgarrado, para que la infamia no se prolongue.

Cuando salía a trabajar, los niños quedaban solos en el cuarto; la esposa de mi otro hermano los cuidaba para que no salieran a la calle. Ella les daba la comida y los llevaba al colegio, pero cuando estaban dormidos, mi cuñado, que estaba dentro de la casa, entraba y abusaba de la niña. ¿Desde cuándo lo hizo? Yo no sé, no quise preguntarle a la niña (Arango y Peñaranda, 2009).

Este penoso episodio al igual que otros tantos que son difundidos constantemente por los medios de comunicación y muchos que quedan en la impunidad por temor, ignorancia, indiferencia o ante la amenaza de los victimarios, deben erradicarse de la historia de tantos niños y niñas que han tenido que sufrir tan infortunados sucesos. Para ello es indispensable influir en la creación de políticas públicas, propuestas educativas, estrategias pedagógicas, experiencias significativas y compromisos reales con la infancia, para reivindicar sus derechos y libertades, asegurando una etapa digna, feliz, segura, libre de circunstancias que les perjudique en su crecimiento, desarrollo, proceso de socialización y acompañados por una sociedad que les cuide, preparándoles para las distintas etapas en el camino de la vida.

Desde dicha perspectiva, el presente artículo tiene por objeto, presentar algunos referentes teóricos y una propuesta educativa que despierte la inquietud frente a la importancia de abogar por 
una cultura, praxis y tecnología que opte por el cuidado de sí como principio en la formación de los niños y las niñas de Colombia.

\section{Acercamiento al concepto de cuidado}

Para definir el concepto es preciso revisar el significado de cuidar. «Cuidar es, por tanto mantener la vida asegurando la satisfacción de un conjunto de necesidades indispensables para la vida, pero que son diversas en su manifestación» (Françoise, 1993, p. 8). Cuidar es encargarse de proteger, dar bienestar; tiene mucho que ver con la interacción entre las personas como parte de la relación humana. Por lo mismo debe ser un proceso cálido, de finura en el trato, compromiso e interés por resguardar de manera especial la vida. A este respecto en los escenarios académicos se ha ido acuñando la expresión «ética del cuidado» desde dos referentes: el primero tiene que ver con un término trabajado por Carol Gilligan al respecto de la moral y la psicología del desarrollo femenino. El segundo está relacionado con la presentación que hace Michael Foucault del cuidado como una tecnología presente en la cultura griega y romana en el período clásico, que permite a los individuos hacer ciertos cambios y transformaciones en sí mismos, con el fin de cultivarse de manera individual.

Sin embargo, también se puede entender como la mejor manera de conducir las acciones tanto individuales como grupales de forma responsable, entendiendo que los contextos sociales permiten la formación de redes de relaciones en las que las personas responden, de una u otra manera, haciendo posible el surgimiento de la responsabilidad y el reconocimiento de las necesidades de los demás; con el fin de vincularse moralmente a dichas causas con comportamientos solidarios y aportar en la medida de las posibilidades a la solución de los problemas de la comunidad.

La ética del cuidado tiene que ver con circunstancias cotidianas de la vida, con despertar el interés por el bienestar, la superviven- 
cia, la autonomía, la justicia, el reconocimiento y cumplimiento de los derechos y deberes. En este sentido, dichos elementos se pueden encontrar en las investigaciones de Jean Piaget (1932) y Lawrence Kohleberg (1981-1984), quienes en sus estudios para describir el proceso y las etapas del desarrollo moral excluyeron inicialmente a las mujeres, concluyendo que ellas tenían menos desarrollo moral que los hombres, puesto que ellas poseen mayor sensibilidad hacia los otros, son capaces de cambiar las reglas; sus juicios se enfocan más hacia la responsabilidad y la moralidad se basa en el cuidado del otro. Por el contrario, los hombres poseen un juicio autónomo y sus decisiones son claras; son individualistas y su moral se basa en los derechos de los individuos.

Los primeros estudios de Piaget sobre el desarrollo moral poco tienen que ver con conceptualizaciones éticas. «Su preocupación se basa en el criterio moral del niño, se dirige a comprender el desarrollo de nociones particulares, como la mentira, el origen de las reglas y del castigo, sin que esto exigiera una comprensión clara de los problemas morales» (Gilligan, 1994, p. 41), Piaget entiende la justicia como el manejo de reglas sociales y el simple acto de transformación de éstas por consenso social. Dichas capacidades cognitivas corresponderían con lo que Kohlberg presenta como principio de un razonamiento convencional. Para la teoría estructural de Piaget y Kohlberg: «es irrelevante contemplar las diferencias en el desarrollo debidas a variaciones culturales, sociales, educativas o de género» (Gilligan, 1994, p. 45). Por tal razón consideran la justicia como el eje central de la moralidad.

Según Gilligan (2004) Kohlberg manifiesta que el desarrollo moral implica un proceso jerárquico de seis etapas, guiado por un sistema racionalista, donde prima la justicia como base fundamental de su teoría, la cual es procedimentalista; o sea, no se puede decir que algo es bueno en general, sólo que la decisión se ha tomado, siguiendo o no las normas. El problema no radica en si los resultados son justos, sino en que se cumplan los procedimientos, para la 
ética de la justicia es necesario partir de las personas como entes separados, independientes, lo cual supone una concepción del individuo como previo a las relaciones sociales, que comprende el mundo como una red de relaciones, en las que se inserta el yo, y surge un reconocimiento de las responsabilidades hacia los demás. En este escenario en el que se analiza el desarrollo moral de los niños y las niñas, las segundas aparecen descalificadas por Piaget y Kohlberg.

\section{Carol Gilligan y la ética del cuidado}

En 1982, Carol Gilligan refuta las afirmaciones de Kohlberg con respecto a las relaciones sociales y a la manera como el yo se inserta, reconociendo las responsabilidades de los demás, a partir de los resultados que obtuvo en estudios realizados con mujeres. En el primer grupo exploró la identidad y el desarrollo moral de los primeros años del adulto, el pensamiento acerca de la moral y la toma de decisiones, y en los otros grupos estudió la relación entre la experiencia el pensamiento y el papel del conflicto en el desarrollo. Gilligan pone de manifiesto que el desarrollo moral de las mujeres es diferente al de los hombres, pero igualmente valioso:

La psicología de las mujeres, que siempre ha sido descrita como distintiva por su mayor orientación hacia las relaciones y la interdependencia, implica un modo más contextual de juicio y un entendimiento moral distinto. Las mujeres dan al ciclo vital un diferente punto de vista en función de diferentes prioridades. (Gilligan, 1994, p. 45).

La autora señala que ellas, en vez de aplicar principios éticos abstractos a cuestiones morales, de conformidad con las teorías tradicionales, emplean estrategias diferentes en la toma de decisiones. La mujer entiende los problemas morales en términos de conflicto de responsabilidades. Su juicio moral sigue una secuencia, en la cual primero se trata de la sobrevivencia, luego de la 
benevolencia y por último de la comprensión reflexiva del cuidado para la resolución de conflictos.

En su primer estudio, presentó una niña y un niño de 11 años, Jake y Amy, reflexionando con respecto a un dilema trabajado anteriormente por Kohlberg:

Un hombre llamado Heinz reflexiona si debe robar o no un medicamento, que no puede comprar, para salvar la vida de su esposa. El farmacéutico se niega a bajar el precio a la medicina, frente a dicha negativa idebe Heinz robar el medicamento? (Gilligan, 1994, p. 51).

Dicho planteamiento permite a Gilligan identificar en las respuestas de sus encuestados las grandes diferencias en la conformación de la conciencia moral. Mientras que Jake hace toda una elaboración lógica en la que confía en la justicia y en medio de su disertación concluye que el juez consideraría justo robar el medicamento, que las leyes son elaboración humana y por lo tanto pueden estar llenas de errores que se deben cambiar. El niño justifica que Heinz robe el medicamento para conservar la vida de su esposa y descarta lo injusto que sería aceptar la negativa del farmaceuta y dejar morir a su ser querido. Esta respuesta de Jake coincide con el razonamiento propio del paso de la niñez a la adolescencia descrito por Piaget por medio del cual afirma: «Es el pináculo de la inteligencia de la niñez, que por medio del pensamiento empieza a descubrir un universo más vasto de posibilidades, naciendo así su capacidad para el pensamiento formal al igual que su capacidad de meditar acerca del pensamiento y de razonar en forma lógica» (Gilligan, 1994, p. 51).

Amy, por su parte, se encuentra en una postura totalmente distinta al dilema, pues para ella robar la medicina es algo errado. Tampoco enmarca el problema con perspectiva matemática desde la lógica, dándole más importancia al bienestar, para ella es importante la 
continua relación del esposo con la esposa, la preocupación de ambos y a la vez comprende la necesidad del farmaceuta. Amy busca la manera de sostener, proteger, cuidar las relaciones, vinculando la salvación de la esposa con la generosidad de hombre que debería rebajar el dinero de la medicina para que la mujer enferma se recuperará:

El mundo de Amy es un mundo de relaciones y de verdades psicológicas, donde una conciencia de la conexión entre personas hace surgir un reconocimiento de las responsabilidades de unas a otras. Los juicios de Amy contienen la vislumbre central de una ética del cuidado mutuo, así como los juicios de Jake reflejan la lógica del enfoque jurídico. (Gilligan, 1994, p. 59).

Es evidente que la niña busca una solución que tome en cuenta las necesidades de todos, considerando que otras personas cuentan con ella y no quiere defraudar a nadie, pues quiere cuidar de todos. Desde esta perspectiva es importante considerar que para las mujeres es muy importante cuidar las relaciones, asumiendo un determinado rol que les permita cargar con responsabilidades que las libre del egoísmo y de dañar a otro.

Por otra parte, Gilligan (2004) entiende la ética del cuidado como la responsabilidad social, desde la que se plantea la búsqueda del bienestar de las personas, con respecto a esto, hace una propuesta desde la cual, aboga por las diferencias, por el reconocimiento de historias particulares, por el cuidado y el deseo de bienestar del otro, por la benevolencia como matriz de las relaciones sociales y del juicio ético. En su teoría, reclama por un espacio, donde el otro deba ser reconocido en su particularidad.

\section{Foucault y el cuidado de sí}

El autor presenta una propuesta diferente a la presentada por la feminista Gilligan. Dicha perspectiva se refiere al arte de la existen- 
cia, la cual implica aprender a cuidarse a sí mismo. Para esto inicia toda una indagación por el mundo antiguo, buscando elementos que permitan descubrir la noción del cuidado en el acontecer de la cultura griega. Al desarrollar una historia del cuidado y de las técnicas de sí, presenta una historia de la subjetividad ${ }^{1}$. De esta manera retoma la cuestión del gobierno de sí mismo en su articulación de las relaciones con los otros. Dicha historia de las primeras formas del cuidado abarcan desde el siglo $\mathrm{V}$ a. $\mathrm{C}$ hasta el siglo $\mathrm{V}$ d. C, período en el que se pueden distinguir tres momentos: el momento socrático, la edad de oro de la cultura del cuidado y el paso del ascetismo al cristianismo.

Con respecto al momento socrático ubicado en el siglo $\mathrm{V}$ a. C. Foucault retoma el diálogo del Alcibíades y la Apología socrática como referentes para presentar la ética del cuidado. En el primero, describe la manera como el principio délfico gnothi seauton «conócete a ti mismo»² fue ganando más importancia en Occidente que epimeleia heutou «cuidado de sí» debido a que los neoplatónicos de los siglos III y IV a.C, al querer organizar los diálogos platónicos

1 Con la expresión subjetividad o subjetivación, Foucault lo que ha querido adelantar es una historia del sujeto o, más bien, de lo que él denomina modos de subjetivación. Es necesario tener en cuenta que dicha historia del sujeto cambia de estilo, de objetos y de metodología a medida que Foucault se desplaza de la cuestión de la episteme al dispositivo y, finalmente, a las prácticas de sí mismo. De esta manera Foucault es conducido hacia una historia de las prácticas en las que el sujeto aparece no como instancia de fundación sino como efecto de una constitución. Los modos de subjetivación son precisamente las prácticas de constitución del sujeto. (Castro, 2004, p. 333).

2 Defradas propone que "conócete a ti mismo» gnothi seauton no es en absoluto un principio de autoconocimiento. Según este autor, esos tres preceptos délficos serían imperativos generales de prudencia: de «nada en exceso» en los pedidos, las esperanzas, ninguna demasía, tampoco en la manera de comportarse; en cuanto a las cauciones, era un precepto que prevenía a los consultantes contra los riesgos de la generosidad excesiva; y con respecto al «conócete a ti mismo», sería un principio según el cual hay que recordar sin cesar que, después de todo, uno no es más que un mortal y no un dios, y por lo tanto no debe presumir demasiado de su fuerza ni enfrentarse con las potencias que son las de la divinidad. (Foucault, 2001, p. 19).

ItINERARIO EDUCATIVO • AÑo XXV, N. ${ }^{\circ} 58$ • 25-45 • JULIO-DICIEMBRE DE ZOII 
dieron un gran valor al Alcibíades considerándolo como el primero, por el que se debería iniciar la lectura y estudio de la obra de Platón. Más adelante: «En el siglo II Albino dijo que cada joven dotado que quisiera mantenerse alejado de la política y practicar las virtudes, debía estudiar el Alcibíades. Este diálogo servía de punto de partida y de programa para toda la filosofía platónica» (Foucault, 1990, p. 55). Es importante aclarar que en dicho texto, Sócrates da un consejo de prudencia al joven Alcibíades al invitarlo a conocerse a sí mismo antes que pretender convertirse en gobernante de Grecia, como principio de la más básica razón, por tanto es necesario resaltar que fueron las acciones de los neoplatónicos y del monje Albino, las que pusieron el «conócete a ti mismo» como el principio que encarna la máxima expresión del pensamiento griego.

La epimeleia heutou «cuidado de sí» quedó relegado, y con dicha noción se fue al olvido una de las reglas más importantes y generales dentro de la cultura griega, la cual es presentada en la Apología socrática como una de las más importantes tareas de Sócrates para con los jóvenes de su tiempo, tal como queda expresado en la defensa que hace de su papel como filósofo y guía de los estudiantes de su tiempo, pues de manera serena responde que se siente orgulloso de la manera como ha llevado su vida y además afirma que no dejará de increpar a los atenienses para que dejen de ocuparse de las cosas que comúnmente despiertan su atención, como la reputación o la fortuna, pero no ellos mismos, diciéndoles:

Y no te ruborizas al poner cuidado en tu fortuna y honores, pero en lo que se refiere a tu razón y a tu alma, no te inquietas por ellas. Si alguno de vosotros contestara, que las cuida, su alma, la verdad y la razón, no creáis que voy a dejarlo e irme de inmediato; no, lo interrogaré, lo examinaré, discutiré a fondo (Platón, 1982, p. 156).

Con estas palabras, Sócrates deja claro al tribunal, que su principal ocupación en la ciudad consiste en filosofar y exhortar a los atenien- 
ses para que no dejen de ocuparse de sí mismos, dando cabida a consideraciones que no merecen atención o por lo menos no tanta como para olvidarse de sí. De esta manera se devela la manera en que para los griegos dicha ocupación de sí, era una labor continua, pero no simplemente como una condición de acceso a la filosofía, sino como un principio de conducta racional que en cualquier forma de vida activa se convirtiera en una premisa de accionar moral. Asimismo se dejan entrever los elementos y movimientos que conforman la noción de cuidado de sí, partiendo de un cambio de mirada desde los otros y el mundo hacia sí mismo, con el fin de generar unas prácticas que tengan por principal tarea la atención del individuo, las cuales son enunciadas por Foucault como las tecnologías que permiten dilucidar la manera en que el individuo actúa sobre sí mismo y las cuales consisten en el descubrimiento de los juegos de verdad, relacionados con las técnicas para entenderse a sí mismo.

\section{Las tecnologías del cuidado de sí}

Las prácticas del cuidado, fueron presentadas por Foucault en las Tecnologías del yo, como aquellos dispositivos que permiten que aflore la subjetividad a partir del cuidado de sí. Existen cuatro tipos principales de estas tecnologías: «Tecnologías de la producción, tecnologías de sistemas de signos, tecnologías de poder y tecnologías del yo, que permiten a los individuos efectuar cierto número de operaciones sobre su cuerpo y su alma, pensamientos, conducta, obteniendo una transformación de sí mismos» (Foucault, 1990, p. 48). Dichas tecnologías no operan por separado, se entrelazan en todo momento, permitiendo abordar la noción de cuidado de sí. De esta manera, aparece la relación entre estar ocupado consigo mismo y la pedagogía, posteriormente la relación entre el cuidado de sí y el conocimiento y en cuarto lugar la relación entre el cuidado de sí y el amor filosófico o relación con un maestro. Estos cuatro problemas con respecto a la noción de cuidado, revelan unas ciertas prácticas que ponen de manifiesto unas tecnologías del yo presentes en la historia del cuidado. 
Estas tecnologías son descubiertas por Foucault a lo largo de la historia de la antigüedad clásica, en los períodos helenísticos e imperiales. Los estoicos proponían retirarse para estar a solas y pensar en sí mismos, dicha manera de actuar se entendía como un ocio activo que implicaba estudiar, leer, prepararse para el futuro o la muerte. Aparece también la relación pedagógica como una tecnología que consiste en que el maestro habla mientras el discípulo permanece en silencio, para luego pensar en lo que ha escuchado y a la vez escuchar la voz que está en su interioridad. Otro elemento fundamental corresponde al examen de conciencia, referido a la manera como cada quien se mira a sí mismo para purificarse antes de dormir.

Seguidamente aparecen otras tres tecnologías; askesis que consiste en ponerse en una situación difícil por medio de la imaginación con el fin de verificar si se es capaz de afrontar tales acontecimientos. La segunda tecnología hace referencia a la gimnasia, la cual consiste en el entrenamiento frente a una situación real y tiene por finalidad probar la independencia del sujeto con respecto del mundo exterior, medir las fuerzas y considerar hasta dónde se puede llevar al individuo por medio del entrenamiento. La tercera está relacionada con la interpretación de los sueños, consiste en meditar las acciones y pensamientos anteriores y posteriores al sueño, para comprender su origen, significado y relación con el futuro.

El contacto con las prácticas propias de la antigüedad, permitió a Foucault realizar una exploración sobre las nuevas formas de subjetividad mediante la indagación por el modo en que el ser humano se convierte a sí mismo en sujeto. Esto lo llevó a identificar y examinar el principio de cuidado de sí, formulado por la filosofía griega y grecorromana. Así las cosas, la preocupación por el cuidado y las técnicas de existencia, expresarían una autonomía por conquistar, pues invitan a una práctica de sí y de la verdad en la que se busca la liberación del sujeto. En consecuencia, sería posible la existencia de un sujeto verdadero desde la proclamación 
de la subjetividad, en otras palabras, el sujeto se autoconstruiría con la ayuda de las técnicas de sí, en lugar de ser constituido por técnicas de dominación del poder o por técnicas discursivas del saber. De hecho, al asumirse a sí mismo como su propia obra de arte, el yo gozaría de autonomía, liberándose de la idea de que entre la moral y la sociedad hay un vínculo necesario. De esta manera el yo se convierte en la nueva posibilidad estratégica para llevar a cabo las prácticas de la libertad, mediante las cuales el individuo busca constituirse y transformarse a sí mismo.

\section{Nel Noddings y el cuidado}

Detenerse en la propuesta de la filósofa Noddings ${ }^{3}$ resulta fundamental debido a la manera en que vincula a las propuestas de Gilligan argumentos sobre la manera de integrar la ética del cuidado con la cuestión educativa. Por tanto la tesis central de su planteamiento consiste en cuidar y ser cuidado como necesidades fundamentales del ser humano. Así, dicho concepto se extiende a los siguientes dominios que deben estar presentes en la educación: cuidado del propio ser, por personas cercanas, extrañas, a los animales, plantas, el mundo y a las ideas. En consecuencia, las fortalezas e intereses de los estudiantes sólo pueden ser desarrollados en un ambiente de cooperación. De todo ello se deriva que la educación moral debe producir personas morales, pero lo más importante es que debe ser moral en sus propósitos, políticas y medios.

3 A partir de la propuesta de Gilligan, en 1984 la filósofa Noddings combinó el reconocimiento de la ética con perspectivas sobre el desarrollo moral de la mujer, y centró sus ideas en el valor de los ciudadanos y la actitud solícita, que se encuentra desde el momento del nacimiento, cuando las personas son totalmente dependientes de los demás. Sostiene Noddings que los enfoques de Piaget y Kohlberg se establecieron a partir del análisis de la personalidad masculina, privilegiando la justicia y la racionalidad. Sin embargo, las mujeres suelen privilegiar otros valores como los de relación, responsabilidad y cuidado. El enfoque de la filósofa nace de una firme convicción feminista en la que defiende la creación de un ambiente de apoyo y solidaridad en instituciones educativas que permitan promover valores que trasciendan la generosidad.

ItINERARIO EDUCATIVO • AÑO XXV, N. ${ }^{\circ} 58$ • 25-45 • JULIO-DICIEMBRE DE ZOII 
De igual manera es importante considerar, desde la perspectiva de Noddings, que desde hace siglos ha habido una gran discusión sobre lo que mueve al ser humano a actuar correctamente en cuanto al bien y el mal, por eso ha centrado sus esfuerzos en una pedagogía que enseñe a pensar correctamente. Uno de los aspectos que más preocupa a Noddings se refiere al cuidado fuera de las consideraciones morales como sucede con la justicia representada por Kohlberg y la tradición kantiana pues esto genera el empobrecimiento de la vida humana. Por tales razones, se ha puesto en tela de juicio que sea posible fundar la ética sobre la razón, ya que ésta es incapaz de proveer la motivación moral para la acción. En el caso de lo expresado por Noddings es el sentimiento el que conduce a la acción.

La reflexión feminista actual ha explorado el camino del sentimiento, desde donde se trata de educar en el cariño y el cuidado como bases fundamentales para obtener la motivación y la perspectiva desde la cual comprometerse por una vida humana plena. Nel Noddings ha estructurado la propuesta femenina de la ética del cariño para la escuela:

Pienso que una ética construida sobre el cuidado es característica y esencialmente femenina, no significa, por supuesto, que no pueda ser compartida por los hombres, de la misma manera que podríamos decir que los sistemas morales tradicionales pueden ser asumidos por las mujeres, pero esta ética se genera a partir de nuestra experiencia como mujeres (Noddings, 1984, p. 8).

Lo primero es aclarar que por «femenina» se entiende la propuesta que nace de la experiencia que históricamente la mayoría de las mujeres han vivido: ser madres y esposas a cargo del hogar, por lo tanto, no se trata de un rasgo esencial -algo exclusivo de la constitución de la mujer-sino histórico: «...ellas fueron confinadas a las actividades que requerían receptividad, relación y sensibilidad...» (Noddings, 1984, p. 2). Las mujeres cuidan, no porque 
haya algo esencial en ellas que las lleve a hacerlo, sino porque han disfrutado y sufrido una experiencia en la que el cuidado es central. En ese sentido, Noddings está a favor de un balance de la experiencia femenina y masculina en la vida humana que pueda abrir nuevas posibilidades para los hombres, las mujeres y la sociedad, generando una experiencia abierta a todo ser humano, especialmente a aquellos que están en posición de cuidar y educar a otros: «La esperanza es que los dos sexos puedan aprender a cuidar» (Noddings, 1984, p. 19).

El cuidado es una cualidad de la relación entre dos personas en donde una es la que cuida y la otra es la que responde a ese cuidado, dicha característica diferencia la ética del cuidado de otros enfoques éticos que suman una imagen del humano como un solitario que debe construir puentes para vivir con otros. Además no concibe el cuidado como una virtud personal que se puede cultivar, como la honestidad o la responsabilidad, es un atributo de la relación que requiere educación y compromiso. En los niños pequeños esta relación es básicamente unilateral, es decir, el adulto-educador cuida y el niño acepta y responde a ese cuidado. A medida que la persona va creciendo va lográndose una relación más recíproca hasta llegar a la relación de adultos, donde los roles del que cuida y el que es cuidado se alternan de acuerdo con las circunstancias y necesidades; pero no será posible llegar a este nivel de adultez si no se ha tenido la experiencia de haber sido cuidado. De ahí la importancia de establecer esta relación desde la infancia. Según Noddings (1992) el cuidado requiere atención y desplazamiento motivacional. Lo primero puede definirse como sentir con el otro y sentir lo que siente. Lo segundo es sentir que la energía que motiva, se moviliza hacia otros y a sus proyectos.

Noddings es consciente de que lograr establecer relaciones de cuidado con todos no es humanamente posible. Habrá personas con las cuales no se logre, pero que necesitan cuidado. ¿̇Qué hacer en dicha circunstancia? Para ella estos casos exigen el ideal 
ético que surge de la memoria de los momentos en que se ha recibido cuidado y permite responder con cariño incluso cuando el sentimiento natural de cuidado no aparece. En cuanto a la ética del cuidado como enfoque de pedagogía moral, dice que la meta más importante de la escuela es lograr que los niños y las niñas se sientan queridos/as y por tanto crezcan llegando a ser personas cariñosas, que aman y son amadas:

...la primera tarea de la escuela es el cuidado de los niños. Deberíamos educarlos a todos para el cuidado y no únicamente para la competencia. Nuestra meta debería ser fomentar el crecimiento de gente que cuida, amorosa y que es posible amar (Noddings, 1984, p. 10).

Noddings propone organizar la escuela a partir de cuatro estrategias, las cuales implican reformar el currículo, el manejo de las clases, la distribución del tiempo, los recursos y especialmente la manera como la relación estudiante-profesor es concebida. Para esto propone la eliminación de las barreras que se han creado para alejar a los estudiantes; de esta forma los educadores podrán asumir su rol de nutrir el ideal ético del cuidado, ya que éste sólo podrá realizarse en un ambiente donde los niños y las niñas se sientan invitados a hacerse seres humanos que cuidan. Esto implica que los educadores asuman un papel activo en el que puedan:

Sugerir, persuadir, inspirar, fomentar, negociar acuerdos, ofrecer ayuda concreta. Sobre todo deben acercarse al estudiante en diálogo, de manera que se construyan buenas decisiones. No respetamos a los estudiantes cuando los dejamos solos para tomar decisiones con base en el capricho. (Noddings, 1984, p. 157).

Educar en el cuidado implica alejar la permisividad, para asumir una postura cooperativa centrada en el respeto y el fomento de la iniciativa personal: «Como educador, el que cuida tiene dos gran- 
des tareas: ampliar el mundo de los estudiantes presentándoles una selección efectiva y a la vez nutrir su ideal ético» (Noddings, 1984, p. 178). Para que esta manera de educar sea viable y efectiva, Noddings propone cuatro aspectos, modelar, dialogar, confirmar y practicar como las estrategias pedagógicas que puede emplear el educador para lograr afianzar el ideal ético de sus estudiantes.

1. Modelar: el educador se convierte en un testimonio de relaciones de cuidado con sus estudiantes. Este ejemplo es clave ya que esta es una pedagogía en donde se aprende viviendo.

2. Diálogo: el diálogo abierto y honesto es vital para desarrollar relaciones de cuidado. Esto implica que los educadores sean capaces de escuchar y responder a las necesidades y sentimientos de los estudiantes. Educadores y educandos discuten honesta y abiertamente lo que les concierne.

3. Confirmación: significa aceptar que los educandos están en una búsqueda sincera de sí mismos. Esto requiere que los educadores atribuyan el mejor motivo posible a las acciones de los estudiantes de acuerdo con las circunstancias. No se trata de alabar a los estudiantes ingenuamente, sino de llegar al fondo de las motivaciones: «el docente debe ver las cosas -actos, palabras, consecuencias- por lo que son, no debe ser tonto, pero puede tratar de acoger con la seguridad de que lo que fue un error podría haber ocurrido por un motivo decente.» (Noddings, 1984, p. 123). Esta estrategia implica cambiar la actual mentalidad docente que parte de una desconfianza natural hacia el estudiante y sus motivaciones. La confirmación de un educador a un educando requiere la clase de sensibilidad y respeto que sólo se puede lograr en una relación de confianza donde se conozcan los motivos y los hechos.

4. Práctica: aprender haciendo. El cuidado se aprende cuidando, por lo tanto hay que posibilitarles a los estudiantes oportu- 
nidades para que, en la medida de su crecimiento, puedan también aprender a cuidar de otros. Los programas de servicio a la comunidad podrían orientarse desde esta perspectiva. En un país que se debate en la violencia y el desprecio por la vida, este enfoque puede brindar posibilidades para reconstruir las relaciones sociales.

Los elementos propuestos por Noddings con respecto a la pedagogía y la práctica del cuidado son innovadores en cuanto vinculan estrategias que propician la transformación de los paradigmas educativos.

\section{Cuidado de sí, una tecnología viable en el escenario educativo colombiano}

Definitivamente, es fundamental que se genere un cambio de mentalidad, que permita a las personas en general y al sector educativo en particular enseñar, asumir, pensar en una actitud de cuidado para con la ciudadanía y en especial para la población infantil. Quizá es el momento de empezar a girar la atención del conocer al cuidar, tal como lo sugirió el pensador francés Michael Foucault, con el fin de descubrir los rasgos más legítimos del pensamiento griego y entender que es probable que conocerse a sí mismo es una difícil tarea. Sería necesario empezar por aprender a cuidar como el camino más seguro para conocerse. Esto, teniendo en cuenta que no es una tarea fácil, pues la tradición occidental se ha montado a partir del conocimiento como el máximo estandarte al que aspiran las personas.

En esta medida es fundamental asumir el cuidado presentado por Foucault, como una legítima práctica de la libertad, en tanto ha dedicado gran esfuerzo teórico a dilucidar el valor y significado del cuidado como una posibilidad de generar transformaciones en sí mismo, lo cual según el pensador es: «un fenómeno bastante importante en nuestras sociedades, desde la época 
grecorromana» (Foucault, 1999, p. 395). Lo más importante en dicho proceso, tiene que ver con que dichas tecnologías generan necesariamente procesos de liberación al interior de la persona, por medio de los cuales permite que aflore su individualidad y se convierta en la medida de sus capacidades, rompiendo con estructuras de poder que al estar alrededor del sujeto le generan un peso que no se siente capaz de soportar. Por tal razón, las prácticas del cuidado, vistas como un proceso de liberación permiten que el sujeto se reconcilie consigo mismo, reencontrándose con su naturaleza y estableciendo una nueva y mejor relación consigo mismo.

En este punto es necesario resaltar que el filósofo francés establece una buena diferenciación entre práctica de liberación y prácticas de libertad. Las primeras están referidas a la manera en que un pueblo se emancipa de sus opresores, lo cual no indica que esté desarrollando prácticas de libertad. Ésta se logra por medio de una actitud ética, en tanto que la ética es la práctica reflexiva de la libertad. De esta manera, al quedar enunciada la relación entre libertad y éticas, es preciso puntualizar que los griegos problematizaban su libertad, para convertirla en un problema ético, viendo el ethos como una manera de conducirse.

Por tanto la libertad para los griegos asumida como la no esclavitud, es entendida como un problema político, en tanto que ser libre también significa no ser esclavo ni de sí mismo ni de los instintos. Es importante afirmar que también se convierte en una manera de cuidar a los demás por medio del libre ejercicio de gobernar, es decir, custodiar la ciudad para sí mismo, pero también para los demás. Desde esta perspectiva el poder no se convierte en la posibilidad de oprimir al otro sino de prestar un servicio que beneficia a todos los miembros de la polis. Así mismo, si una persona sabe quién es, de qué es capaz, sabe conducirse en su casa, frente a todas sus cosas, entonces sabe cuidar de sí y por lo mismo ejerce las prácticas de la libertad. 
Precisamente una generosa formación en las prácticas de la libertad es la que necesitan los niños de Colombia y los ciudadanos en general con el fin de saber trabajar para el bienestar y cuidado de los demás rompiendo con aquellas absurdas relaciones de poder dedicadas a inflar egos, promover jerarquías, manipular a las gentes y poner en riesgo el bienestar de la población. Para esto es necesario comprender que se requiere la disposición para dejar que el otro exprese su propia razón, palabras y subjetividad, sin querer coartar su libertad, por otra parte esa gran tarea de educar en el cuidado puede ser apoyada por los aspectos propuestos por Noddings y que tienen que ver con modelar, dialogar, confirmar y practicar como las estrategias pedagógicas que conducen a la idea ética de cuidado, la cual se encuentra en su mayoría en las manos del maestro quien orienta y acompaña desde y para el cuidado, haciéndose partícipe de la formación de un ser humano que sabe vivir las tecnologías que lo hacen libre.

Finalmente es importante recordar las siguientes palabras:

El ethos que cuida y ama es terapéutico y liberador. Sana llagas, despeja el futuro y crea esperanzas. Con razón dice el sicoanalista Rollo May: «En la actualidad confusión de episodios racionalistas y técnicos, perdemos de vista al ser humano. Debemos volver humildemente al simple cuidado. El mito del cuidado, sólo él, nos permite resistir al cinismo y a la apatía, dolencias psicológicas de nuestro tiempo» (Boff, 2003).

\section{Bibliografía}

Boff, Leonardo. (2003). Tomado de la columna semanal de Leonardo Boff. 26 de julio de 2003. En www.servicioskoinonia.org/boff

Castro, Edgardo. (2004). El vocabulario de Michael Foucault. Prometeo 3010, Universidad de Quilmes. 
Foucault, Michael. (1990). Michel Foucault, Tecnologías del yo y otros textos afines. Barcelona, Paidós Ibérica, S.A de la Universidad Autónoma de Barcelona.

Foucault, Michael. (1999). Estética, ética y hermenéutica. Introducción, traducción y edición a cargo de Ángel Gabilindo. Obras Esenciales, Vol. III, Barcelona: Paidós.

Françoise Colliére, Marie. (1993). Promover la vida. Madrid: McGraw-Hill.

Gilligan, Carol. (1994). La moral y la teoría. Psicología del desarrollo femenino. Traducción de Juan José Utrilla. México: Fondo de Cultura Económica.

Noddings, Nel. (1984). Caring: a feminine approach to ethics and moral education. Berkeley: University of California Press.

Noddings, Nel. (2002). Educating moral people: a caring alternative to character education. Nueva York: Theachers College Press.

Noddings, Nel. (1992). Shaping an acceptable child. En Andrew Garrod Ed Learning for life: moral education theory and practice. Westport, Conneticut: Praeget.

Platón. (1981-1982). Apología de Sócrates. Madrid: Gredos.

Quiroz. M., y Peñaranda. F. (2009). Significados y respuestas de las madres al abuso sexual de sus hijas(os). En: Revista Latinoamericana de Ciencias Sociales, Niñez y Juventud. 7, 2.

Recibido $\in$ abril $d \in$ ZOII Arbitrado $\in$ n junio de ZOII 